\title{
Research on Supply Chain Risk Transmission Mechanism Based on Improved SIRS Model
}

\author{
Hongchun Wang and Xinyan Zhang $\mathbb{D}$ \\ Beijing University of Civil Engineering and Architecture, Beijing, China \\ Correspondence should be addressed to Xinyan Zhang; zhangxinyan_zxy@163.com
}

Received 30 July 2021; Revised 14 December 2021; Accepted 29 December 2021; Published 21 January 2022

Academic Editor: Kebing Chen

Copyright ( 92022 Hongchun Wang and Xinyan Zhang. This is an open access article distributed under the Creative Commons Attribution License, which permits unrestricted use, distribution, and reproduction in any medium, provided the original work is properly cited.

\begin{abstract}
An improved SIRS model is constructed as a supply chain risk propagation model, based on the actual situation that node enterprises are eliminated in the supply chain network. From the perspective of the small-world network, simulation is carried out to analyze the risk transmission mechanism in the supply chain network and the influence of various parameters on the supply chain risk transmission and the node enterprises in the supply chain network. Research shows that the process of eliminating node enterprises is also a process of supply chain network's adjustment and gradual stability. In order to reduce the negative impact of this process on the supply chain network, node enterprises should take effective measures based on their own conditions to improve risk prevention capabilities, coordinate risk transmission, and enhance risk immunity, so as to improve the resistance of the entire supply chain to risks.
\end{abstract}

\section{Introduction}

With the characteristics of innovation, synergy, win-win, openness, and environmentally friendly, the supply chain can promote cross-border and synergistic development of industries and is also an important carrier to lead globalization to enhance competitiveness. The application of supply chain network is becoming more and more widespread due to the advancement of science and technology, the policy guidelines, and the trend of cooperation development among enterprises. The possibility of being affected by risks also increases as the supply chain industry grows and develops. External factors such as natural disasters, public health emergencies, economic crises, and policy regulations and internal factors such as inadequate work quality of personnel, poor management system, and difficulties in capital turnover may become risk factors that induce problems and negatively affect the nodes in the supply chain, which in turn leads to risk transmission through the supply chain network path and further spread of harm. Supply chain risks can have an impact on the normal operation of the supply chain network and affect the development status of each node enterprise in the supply chain network. As global climate change scenarios have increased in recent years and climate effects have intensified, many supply chain companies must reassess the impact of supply chain risks due to natural disasters. The outbreak of COVID-19 in 2020 has left global automotive, machinery, communications, and other manufacturing sectors facing parts shortages, threatening supply chain disruptions within weeks and forcing delays in the launch schedule of new products. On 06 February 2020, Nintendo, Japan, announced that the new version of switch will be delayed on sale because of the impact of the outbreak. On February 8 2020, Apple Inc. issued a financial warning on first quarter operating income due to the impact of the epidemic. The disruption shock to China's manufacturing sector generated devastating impacts on the global supply chain, as China is the largest supply base in the world. A survey in February 2020 shows that $94 \%$ of the Fortune 1000 were experiencing supply chain disruptions [1]. Therefore, supply chain risk has become a major factor limiting the development of supply chain management model and an important issue that companies have to consider in the implementation of supply chain management. 
In 2017, the General Office of the State Council issued the "Guidance on Actively Promoting Supply Chain Innovation and Application" to improve global supply chain security, encourage enterprises to establish supply chain risk warning systems for important resources and products, develop and implement supply chain security plans, enhance supply chain risk management, make improvements to global supply chain risk warning mechanisms, and increase supply chain risk prevention and control capabilities [2]. It is essential to accurately grasp the supply chain risk transmission mechanism and analyze the factors affecting the transmission of supply chain risks to grasp the law of supply chain risk transmission, target the supply chain risk early warning, stop the transmission of risks in the supply chain network in time, and minimize the threat and loss brought by risks to the supply chain. In the actual process of supply chain in dealing with risks, there is a possibility of elimination for the enterprises in the chain. Therefore, it is necessary to consider the study of supply chain operation where elimination exists, so as to more accurately understand the mechanism of supply chain risk transmission and supply chain changes. Based on the above background, considering the possibility that the network nodes are eliminated from the network by death after infection, an improved SIRS model is proposed in this paper, which is numerically analyzed based on the small-world network. Thus, the supply chain risk transmission mechanism is analyzed to provide a reference for defending the risk propagation into the supply chain network.

\section{Literature Review}

2.1. Supply Chain Risk Transmission. The study of supply chain risk transmission originated from the study of risk transmission among enterprises in the early days. With the gradual increase of linkages between enterprises, supply chain networks developed along the way. The study of risk dynamics among enterprises further evolved into the study of supply chain risk. Jüttner et al. noted that the issue of supply chain risk has attracted attention, exploring practitioners' perceptions of supply chain risk and supply chain risk management strategies [3]. Hassini et al. presented a conceptual framework using the P-A concept for analyzing the complexities of supply network risk, with particular emphasis on the role of network relationships, and then pointed out that the elements of supply chain risk transmission include four major components, namely, preconditions, risk events, transmission paths, and impact generation [4]. Cheng and Liu classified the supply chain risk transmission mode into five types: forward chain transmission mode, reverse chain transmission mode, radial network transmission mode, centralized network transmission mode, and interactive network transmission mode [5]. Li believed that supply chain risk conduction included four basic components, namely, risk source, transmission medium, transmission node, and risk recipient, and then combined these four elements to analyze the supply chain risk conduction process [6]. Li analyzed supply chain risk transmission from a theoretical perspective, subdivided it into five modes, and used the risk mode as the basis for risk identification, laying a foundation for supply chain risk assessment and decision-making [7]. Chen, Ni, and Tang believed that the demand transmission process management was an important part of supply chain management, using the features of fractal theory to build the fractal reengineering of supply chain demand process [8]. These studies adopt a qualitative approach to study supply chain risk transmission from a global perspective; clarify the influencing elements, propagation patterns, and other characteristics of risk transmission in the supply chain; and give some suggestions on how to deal with risk transmission.

With further research, some quantitative analysis methods have been applied to the study of supply chain risk transmission. The research perspectives have also been further developed. In addition to analyzing the risk transmission of the supply chain as a whole, some scholars have also studied the propagation of risk in the supply chain of a certain industry. Cheng and Liu constructed a supply chain risk transmission path model and used mathematical means to describe the boundary conditions and flow values of risk bursts, reflecting the actual situation of supply chain risk transmission [9]. Shi et al. used the Bayesian network approach to analyze the changes in the probability of supply chain risks during transmission [10]. Jiantong et al. used structural equation modeling to analyze a quantitative survey conducted in 31 Chinese automotive-related companies, revealing that the disruption risk of a supplier is believed to affect its manufacturers and is further found to exert adverse influence on its downstream distributors [11]. The enterprises in the supply chain can be abstracted as nodes, with complex connections between the nodes. Therefore, researchers believed that the complex network theory was applicable to the analysis of supply chain networks, which led to the research of supply chain-related problems based on complex networks [12]. Based on the study of the complexity of supply chain network, Wang et al. analyzed the complexity of supply chain network structure in the context of disruption risk and proposed a quantitative measurement method for the complexity of supply chain network based on DSM model [13].

The existing studies on supply chain risk transmission are relatively more analyzed by qualitative methods, which can reflect the characteristics of risks in the supply chain network relatively easily. With the gradual improvement of research, some quantitative analysis methods are used to analyze the influencing factors and probability of occurrence of supply chain risk transmission. The introduction of the concept of complex networks allows supply chain networks to be described more concretely, providing a theoretical basis for analyzing the regular characteristics of risk transmission in supply chain networks.

2.2. Epidemic Models for Supply Chain Research. To more accurately reflect the characteristics of risk transmission in complex supply chain networks, system dynamics research approach has been considered for introducing supply chain risk transmission studies. The epidemic model belongs to a 
classical transmission dynamics model being widely used not only in infectious disease research, but also in risk transmission research in multiple fields. Some scholars have analogized the transmission of infectious diseases to supply chain risk transmission. Thus, SIS, SIR, and SIRS infectious disease models are used to conduct supply chain risk transmission studies, based on complex networks. Based on the idea of virus transmission, Jiang et al. constructed the risk model of supply chain and simulated the risk of communication mechanism between upstream and downstream enterprises and core enterprises [14]. Yang and Zhang established a supply chain network risk propagation model (SIS-RP) to simulate and analyze it from the perspective of the whole supply chain network, which reflects the risk propagation behavior and characteristics in the supply chain network to a certain extent [15]. Lv et al. further revised Yang and Zhang's research and established an SIRSCR model from the perspective of small-world networks, expounding the scope and influencing factors of supply chain risk transmission [2]. Jing used the SIR model to simulate the risk propagation process of the food supply chain, verified the impact of the management capability of the food supply chain on the risk propagation, and proposed a corresponding risk control mechanism for the risk propagation of the food supply chain [16]. Tang drew on the SIRS model to construct a risk propagation model and investigate the propagation mechanism of fresh e-commerce supply chain logistics risks in the network [17]. Based on complex network theory, Wang used epidemic model (SIRS model) to study supply chain risk transmission and discuss the mechanism and evolution of the complex supply chain network risk transmission [18].

In the study of supply chain risk transmission using the epidemic model, existing studies directly apply models such as SIR and SIRS to describe the propagation of risk in supply chain networks, lacking consideration of the actual survival of firms in supply chain networks. Thus, based on the actual situation that nodal enterprises may be eliminated in the supply chain network, this study constructs an improved SIRS model, simulates it from the perspective of small-world network, and analyzes the risk transmission mechanism and the impact of each parameter on the supply chain risk transmission and nodal enterprises in the supply chain network according to the simulation results. The study provides a model reference for analyzing the risk transmission mechanism in the supply chain and its changes on the state of nodes in the supply chain network, reflects the behavior and characteristics of risk propagation in the supply chain network, and provides suggestions for effective control of the spread of risk across the supply chain network.

\section{Model Building}

3.1. Classic SIRS Model. The four most basic models in the compartment model of infectious disease dynamics are SI, SIS, SIR, and SIRS. These several classes of models incorporate three categories of status nodes, $S$ (susceptible), $I$ (infectious), and $R$ (removed), according to epidemiological methods to divide the population, resulting in different transmission models based on the different transitions between the three states. The difference between the SIRS model and other models is that the immunity acquired after cure is not permanent, but there is a certain probability of losing immunity and becoming susceptible again. When some nodes in the network become risky due to virus infection, i.e., infectious $I$, they will transmit the virus to neighboring nodes in the susceptible state $S$ with probability $\beta$ and become infectious $I$. After a period of adjustment, infectious individual $I$ will be cured with probability $\gamma$ and turn into removed individual $R$. However, this immune state is not permanent, and this immunity will disappear with probability $\delta$ as time changes, making it revert to the susceptible state $S$. The change relationships of the nodes in the SI, SIS, SIR, and SIRS models are depicted separately in Figure 1 .

\subsection{Improved SIRS Model}

3.2.1. Model Description. The classical SIRS model reflects a series of processes in the transmission of infectious diseases in which individuals become infected, infectious, cured, immune, and immune incapacitated. In this model, there is only a certain probability that an infected person will be transformed into a removed one after cure. In practice, there is a certain probability that an infected person will not be able to fight off the disease and die, i.e., be "eliminated" by the infectious disease. Therefore, the SIRS model is improved by adding a fourth state node $D$ (died) and the probability of elimination $\mu$. The infection rules are as follows (Figure 2):

(1) After exposure to infected individuals, susceptible individuals will be infected with some probability and change from a healthy state to an infected state.

(2) After a period of adjustment, the infected individual will be cured with a certain probability and return from the infected state to the healthy state, and there exists a certain probability of death, i.e., changing from the infected state to the eliminated one.

(3) Infected individuals will generate immunity of their own after recovering health and become in immune state. However, as time changes, this immunity will disappear with certain probability, and the individual will revert to the state of susceptible to infection.

3.2.2. Model Applicability Analysis. To analyze whether the improved SIRS model is applicable to the study of risk transmission in supply chain networks, it is necessary to determine whether the characteristics of risk propagation in supply chain networks are highly similar to the nature of the improved SIRS model. The analysis is carried out for the characteristics of virus and risk in transmission from three perspectives: transmission range, transmission process, and transmission direction.

First, from the perspective of the scope of transmission, the virus is based on the spread of social groups consisting of independent individuals. The edge of the network formed by people contacting and communicating with each other also 


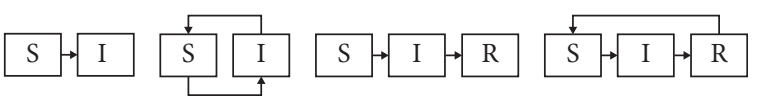

FIGURE 1: Schematic diagram of SI, SIS, SIR, and SIRS epidemic models.

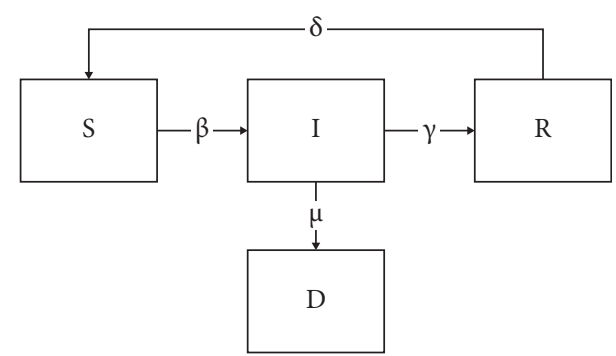

FIGURE 2: Schematic diagram of the improved SIRS model.

constitutes the way of virus transmission. The supply chain network takes enterprises as nodes, and the exchange of resources between enterprises forms the edges of the network. Hence, both the social network of virus transmission and the supply chain network of risk transmission can be regarded as complex networks, and the abstraction of the scope of virus and supply chain risk transmission is described consistently.

Second, from the perspective of the transmission process, the virus is transmitted from an infected person to others through contact with susceptible people. The infected person may die without effective treatment and become eliminated, or he/she may regain health through treatment or autoimmunity and become immune. Over time, an immune person may also lose immunity and become susceptible again. In a supply chain network, companies in risky situations due to risky shocks will transmit the risk to upstream and downstream companies via trading cooperation. If a company infected with risk actively takes effective risk response measures, it may temporarily eliminate the risk and become immune. If companies fail to take timely countermeasures or have limited ability to deal with risks and are unable to maintain their business, they will go out of business and be eliminated from the supply chain network as a result. Companies that have successfully withstood risk shocks and gained immunity are not immune to risk forever. If there are deficiencies in risk management, they may shift to a vulnerable state again and have the probability of suffering from risk shocks again. Therefore, the adoption of an improved SIRS model allows for a description of the process by which supply chain risks propagate and cause impacts.

Third, from the perspective of transmission direction, the virus does not have a specific direction in interpersonal transmission. Although the direction of resource transmission in the supply chain network is from upstream enterprises to downstream enterprises, the spread of risk is radial. Through cooperation and communication, the riskinfected enterprises may transmit the risk to the downstream enterprises as well as the upstream enterprises. The direction of risk propagation in supply chain networks has the same uncertainty as the direction of virus propagation.

According to the above three points and with reference to the related literature of scholars in Section 2 using SIR model and SIRS model to study supply chain risk management, the improved SIRS model has the applicability, feasibility, and research value to study the transmission of risk in supply chain networks adopting the improved SIRS model.

3.2.3. Model Threshold Analysis. The supply chain network can be regarded as a complex network with small-world network characteristics in the context of cloud computing and globalized procurement. The improved SIRS model is described using mathematical means, and the variables and parameters used in the model are defined in Table 1 [19].

According to the infection rule of the SIRS model under the elimination mechanism and

$$
s(t)+i(t)+r(t)+d(t)=1,
$$

four equations are established:

$$
\left\{\begin{array}{l}
s(t+\Delta t)-s(t)=-\beta\langle k\rangle \cdot s(t) \cdot i(t) \cdot \Delta t+\delta \cdot r(t) \cdot \Delta t \\
i(t+\Delta t)-i(t)=\beta\langle k\rangle \cdot s(t) \cdot i(t) \cdot \Delta t-\gamma \cdot i(t) \cdot \Delta t-\mu \cdot i(t) \cdot \Delta t \\
r(t+\Delta t)-r(t)=\gamma \cdot i(t) \cdot \Delta t-\delta \cdot r(t) \cdot \Delta t \\
d(t+\Delta t)-d(t)=\mu \cdot i(t) \cdot \Delta t
\end{array} .\right.
$$


TABle 1: Variables and parameter definitions used in the model.

\begin{tabular}{|c|c|c|}
\hline Symbols & Definitions & Implications for supply chain networks \\
\hline$s(t)$ & $\begin{array}{l}\text { Percentage of individuals in a susceptible state at time t } \\
\text { to the total number of individuals }\end{array}$ & $\begin{array}{l}\text { Percentage of the number of enterprises with the possibility of infection } \\
\text { risk at time } t \text { to the total number of enterprises }\end{array}$ \\
\hline$i(t)$ & $\begin{array}{l}\text { Percentage of individuals in an infected state at time } t \\
\text { to the total number of individuals }\end{array}$ & $\begin{array}{c}\text { Percentage of the number of enterprises exposed to risk at time t to the } \\
\text { total number of enterprises }\end{array}$ \\
\hline$r(t)$ & $\begin{array}{l}\text { Percentage of individuals in an immune state at time } t \\
\text { to the total number of individuals }\end{array}$ & $\begin{array}{c}\text { Percentage of the number of enterprises with immunity to risk at time } t \\
\text { to the total number of enterprises }\end{array}$ \\
\hline$d(t)$ & $\begin{array}{l}\text { Percentage of individuals in an eliminated state at time } \\
t \text { to the total number of individuals }\end{array}$ & $\begin{array}{l}\text { Percentage of the number of enterprises eliminated for failure to } \\
\text { withstand risk at time t to the total number of enterprises }\end{array}$ \\
\hline $\mathrm{B}$ & Probability of infection & Probability of infection risk \\
\hline$\gamma$ & Probability of immunity & Probability of immunity to risk \\
\hline$\Delta$ & Probability of losing immunity & Probability of losing immunity to risk \\
\hline$\mu$ & Probability of being eliminated & Probability of being eliminated \\
\hline$<k>$ & \multicolumn{2}{|c|}{ Network average degree } \\
\hline
\end{tabular}

The equation of the SIRS model in the eliminated state can be written as

$$
\left\{\begin{array}{l}
\frac{\mathrm{d} s}{\mathrm{~d} t}=-\beta\langle k\rangle s i+\delta r \\
\frac{\mathrm{d} i}{\mathrm{~d} t}=\beta\langle k\rangle s i-\gamma i-\mu i \\
\frac{\mathrm{d} r}{\mathrm{~d} t}=\gamma i-\delta r \\
\frac{\mathrm{d} d}{\mathrm{~d} t}=\mu i
\end{array} .\right.
$$

According to formula (1), $d(t)=1-s(t)-i(t)-r(t)$, and the first three equations in formula (3) do not contain $d$. Therefore, formula (3) can be reduced in order, resulting in

$$
\left\{\begin{array}{l}
\frac{\mathrm{d} s}{\mathrm{~d} t}=-\beta\langle k\rangle s i+\delta(1-s-i-d) \\
\frac{\mathrm{d} i}{\mathrm{~d} t}=\beta\langle k\rangle s i-\gamma i-\mu i \\
\frac{\mathrm{dr}}{\mathrm{d} t}=\gamma i-\delta r
\end{array}\right.
$$

Let $(\partial s / \partial t)=0,(\partial i / \partial t)=0,(\partial d / \partial t)=0$, the equilibrium point of the above equation be $(s, i, r)=(1,0,0)$, and $(s, i, r)=(\gamma+\mu / \beta\langle k\rangle, 0,0)$.

Therefore, according to the above analysis, after a period of time in the system, the number of $S$ and $D$ will reach a stable state, and there will be no infected or immunized ones.

\section{Simulation}

4.1. Simulation Analysis of System Development Trend. In order to deeply understand the characteristics of supply chain risk propagation paths, numerical simulations of the improved SIRS model are conducted using Matlab 2020a to verify the model characteristics, analyze the laws of numerical changes, and then grasp the key factors affecting supply chain risk transmission. A small-world network with parameters $N=100,<k \geq 3$, and $p=0.1$ is taken for simulation, and the values of risk propagation parameters of the supply chain are set as $\beta=0.3, \gamma=0.4, \delta=0.1$, and $\mu=0.01$ [20]. The parameters are brought into the model for 30 simulations, and the results are averaged. The simulation results are obtained as shown in Figure 3.

It is known from Figure 3 that the number of susceptible individuals $S$ decreased first, then increased, and tended to be stable with increasing time. The numbers of infected individuals $I$ and immunized ones $R$ increased first, then decreased, and then tended to change toward values of 0 . The number of eliminated individuals $D$ showed an increasing trend and tended to be stable. As can be judged, after a certain period of time, no infected person $I$ or immune person $R$ will be in the system, and the number of susceptible persons $S$ and eliminated persons $D$ reaches a stable value, showing that the simulation results are consistent with the results of mathematical analysis. The simulation results verify the accuracy of the model and reflect the impact of risk transmission on the supply chain after considering the presence of elimination situations.

When there is a risk of propagation in the supply chain network, the companies on some nodes will be eliminated due to insufficient ability to resist the risk. With the development of time, the enterprises infected with risk and immune to risk in the supply chain network will not exist, while the number of eliminated enterprises will continue to grow and eventually reach a stable value. Although there are still some enterprises in the supply chain that are stable in the susceptible state and do not suffer from risk infection, it is difficult for the actual operation of the supply chain system to achieve the expected effect at this point because of the high number of eliminated enterprises, making the risk propagation have a great impact on the development of the industry to which the supply chain belongs.

\subsection{Analysis of the Impact of Risk Elimination Ability on Risk} Transmission. The difference between the improved SIRS model and the classical SIRS model is that the improved SIRS model adds the link of infected person $I$ to eliminated person $D$ as well as introducing the elimination coefficient, which is the probability of occurrence of this link $\mu$ to reflect the risk elimination ability. Risk elimination capability is an important indicator factor of the improved SIRS model. In the supply chain reality, whether supply chain firms will be 


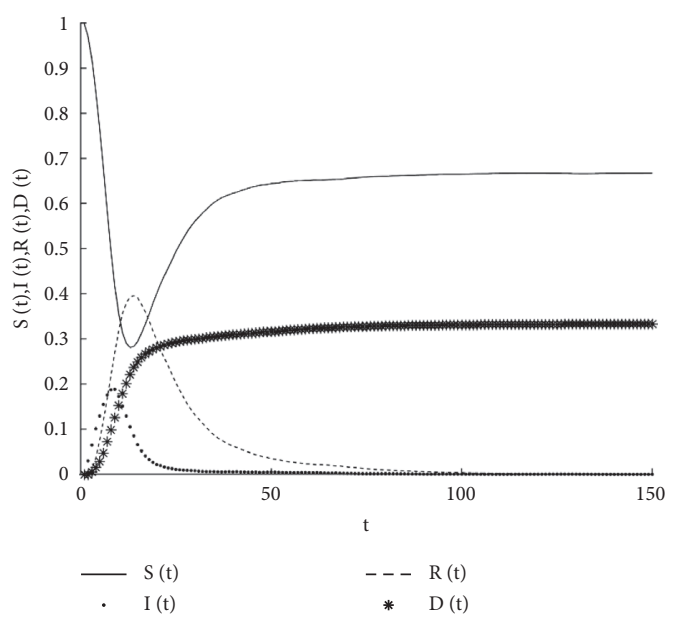

FIGURE 3: Simulation results of SIRS model under elimination mechanism.

eliminated from the supply chain after being infected with risk is importantly related to the series of changes generated by the supply chain facing risk of disturbance. Therefore, the improved SIRS model with different elimination factors is simulated for an in-depth analysis of the impact of risk elimination capacity on risk transmission. The model parameters in Section 4.1 were used, and the values of $\mu$ were changed to $0.001,0.01,0.1,0.3$, and 0.5 .30 simulations were performed for each parameter value, and the results were averaged to obtain the relationship between the percentage of infected nodes $I(t)$, the percentage of eliminated nodes $d(t)$, and the elimination factor $\mu$ in the system, respectively.

According to the analysis in Section 4.1, the number of infected individuals in the system increases to a maximum value and then decreases to zero with the system reaching a steady state. From Figure 4, it can be seen that as the elimination factor increases, the time it takes for the number of infected individuals in the system to reach a maximum and the time it takes for the system to finally reach stability are both reduced. Figure 5 shows that the time to stabilize the percentage of eliminated nodes in the system decreases gradually, and the percentage of eliminated nodes gradually increases. It shows that the increase of elimination coefficient will accelerate the speed of eliminating nodes in the system and increase the number of the eliminated nodes in the system eventually. In this process, although the maximum number of infected nodes is relatively reduced, the increase of elimination coefficient will cause more damage to the system because the eliminated nodes completely lose their value to the system and have no possibility of healing. This shows that when the probability of elimination of enterprises in the supply chain increases, the negative impact on the whole supply chain will increase accordingly. For the supply chain system, reducing the risk of enterprise elimination is significant for maintaining the stability of the supply chain.

4.3. Analysis of the Impact of Risk Infection Ability on Risk Transmission. Risk infectivity refers to the ability of a

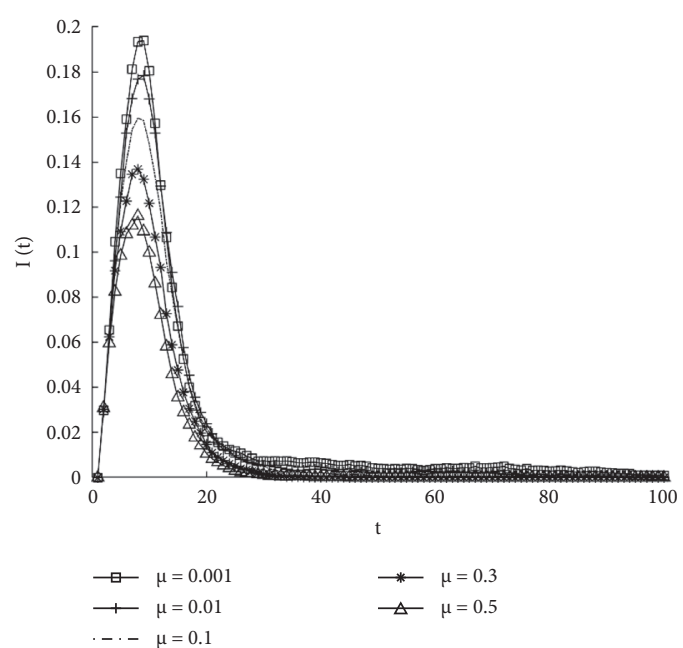

FIgURE 4: The relationship between the percentage of infected nodes and the elimination coefficient.

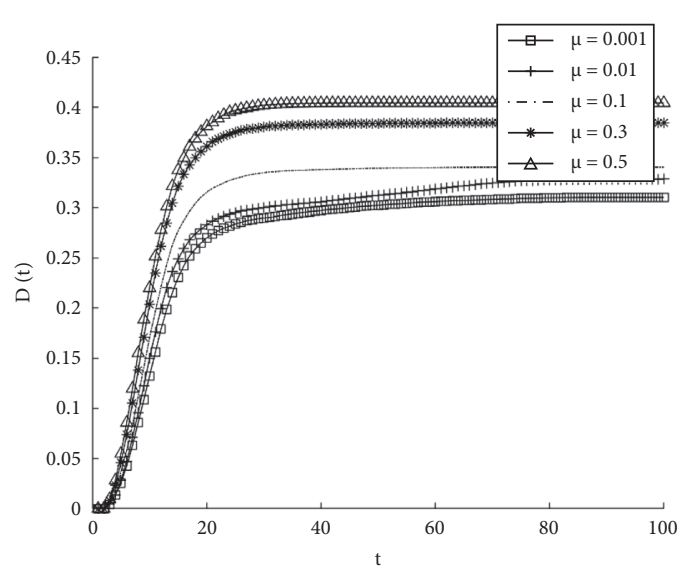

FIGURE 5: The relationship between the percentage of eliminated nodes and the elimination coefficient.

neighboring node in the infected state to infect a node in the susceptible state, making it sick and infected state node, which is expressed by the probability of occurrence $\beta$ of $S-I$ in the model. In the actual supply chain, risk infectivity directly reflects the ability of risk propagation and the prevention effect of enterprise on risk and also has a direct impact on the risk propagation in the supply chain. In order to deeply analyze the influence of risk infectivity on risk transmission, the improved SIRS model with different infection coefficients $\beta$ was simulated. The model parameters in Section 4.1 are used, and the values of $\beta$ are changed to $0.15,0.2,0.25,0.3$, and 0.35 . Each parameter value is taken for 30 simulations, and then the results are averaged. The relationship between the percentage of infected nodes in system $I(t)$, the percentage of eliminated nodes $d(t)$, and the infection coefficient $\beta$ is obtained.

As can be seen from Figure 6, the maximum value of the percentage of infected nodes in the system gradually increases with the increase of the infection coefficient, and the growth rate of infected nodes in the system accelerates. In 


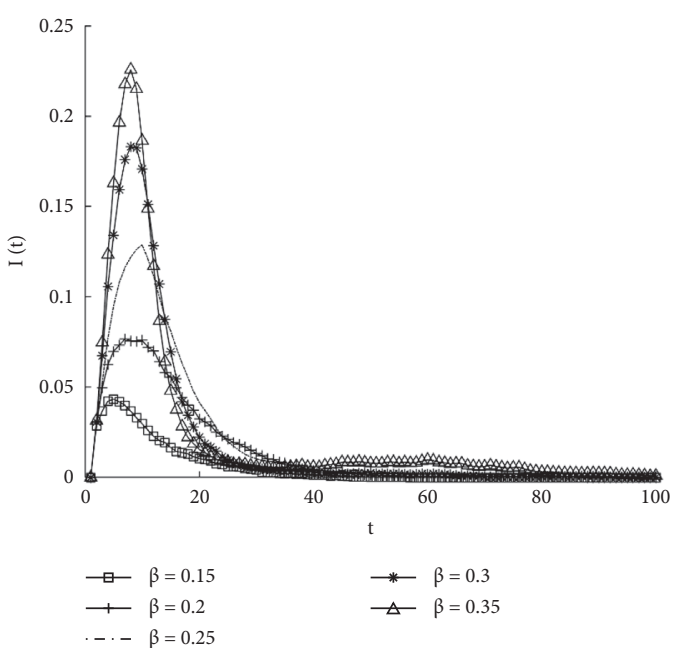

Figure 6: The relationship between the percentage of infected nodes and the infection coefficient.

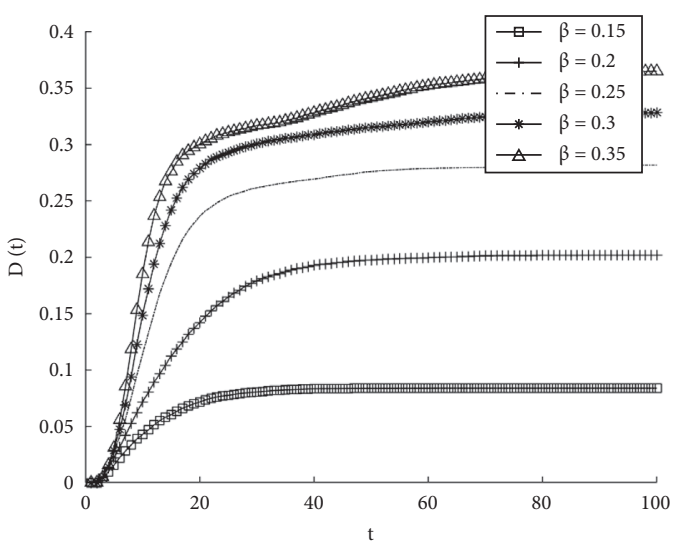

Figure 7: The relationship between the percentage of eliminated nodes and the infection coefficient.

Figure 7, it takes longer time for the percentage of eliminated nodes in the system to stabilize with the increase of the infection coefficient, and the percentage of eliminated nodes gradually increases. This indicates that the increase of the infection coefficient will significantly accelerate the speed and number of infected nodes in the system on the one hand, and it will have an indirect effect on the increase of the number of eliminated nodes on the other hand. In the absence of external interference, the time for the system to stabilize also increases. It can be seen that when the probability of being infected in the supply chain increases, it will have more and more negative impact on the whole supply chain and increase the difficulty of reducing system loss and restoring system stability. It is necessary to consider the reduction of risk infection capacity in the system when maintaining the stability of the supply chain system and reducing the negative impact of risk.

4.4. Analysis of the Impact of Node Resilience on Risk Transmission. Node resilience refers to the ability of an

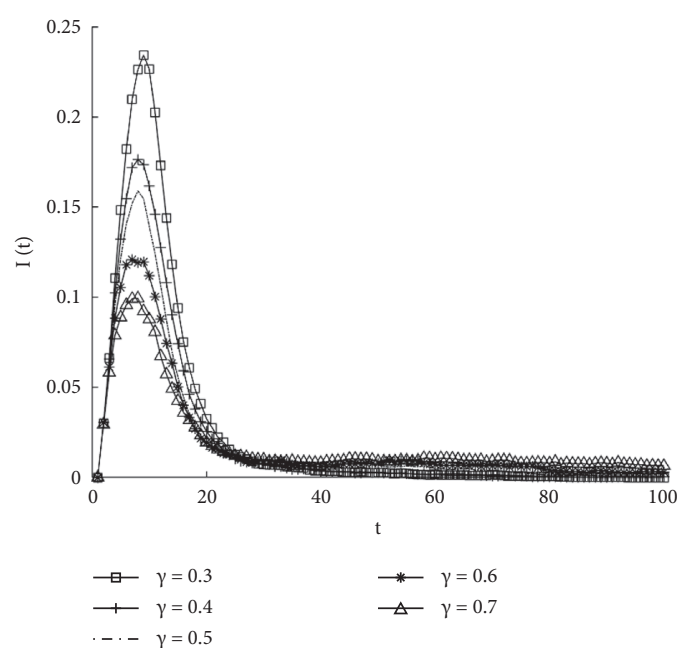

Figure 8: The relationship between the percentage of infected nodes and the immune coefficient.

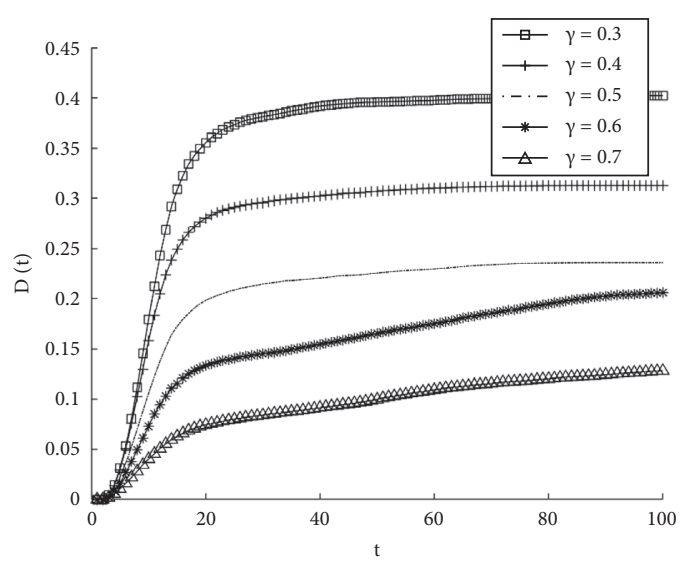

FIGURE 9: The relationship between the percentage of eliminated nodes and the immune coefficient.

infected state node in the system to recover and become an immune one, which is expressed by the probability of occurrence of $I-R$ in the model $\gamma$. In supply chain practice, node resilience directly reflects the resilience effect and the ability of enterprises to recover from supply chain risks, which is important for enterprises to cope with risk injuries. A simulation of the improved SIRS model with different immunity coefficients $\gamma$ is conducted to further analyze the impact of node resilience on risk transmission. The model parameters in Section 4.1 are used, and the values of $\gamma$ are changed to $0.3,0.4,0.5,0.6$, and 0.7 . The simulation is performed 30 times for each parameter value, and the results are averaged. The relationship between the percentage of infected nodes in system $I(t)$, the percentage of eliminated nodes $d(t)$, and the immunity coefficient $\gamma$ is obtained.

As can be seen from Figure 8, with the increase of the immunity factor, the maximum value of the infected node ratio in the system gradually decreases, and the growth rate of infected nodes in the system slows down. As is shown in 
Figure 9, the percentage of eliminated nodes in the system decreases gradually, and the growth rate of eliminated nodes slows down with the immunity factor increasing. This indicates that the increase of immunity coefficient can indirectly alleviate the phenomenon of infected or eliminated nodes in the system, thus reducing the damage of risk to the system. It can be seen that the increased probability of obtaining immunity for companies in the supply chain has a positive impact on the effectiveness of the entire supply chain in coping with risks and also reduces the threat of risks to individual nodal companies. Consequently, the improvement of node resilience in the system should also be taken into account when maintaining the stability of the supply chain system and reducing the negative impact of risks.

\section{Conclusion}

Supply chain risk management plays an important role in preventing risk infringement, responding to risk threats, and maintaining the stability of the entire supply chain network. When facing risks, node enterprises in the supply chain network have the possibility of being eliminated from the supply chain due to their inability to effectively resist the risks. Based on the actual situation that nodal enterprises in the supply chain may experience, the "infected-eliminated" link is added to the SIRS model. An improved SIRS model is constructed to simulate the complex network of supply chain. We analyze the risk propagation paths and effects in the network from a macro perspective and make suggestions for the supply chain to deal with risks. Based on the simulation and analysis, the following conclusions are drawn:

(1) When supply chain risk spreads, there is a possibility that firms will be eliminated. After some enterprises are eliminated, the supply chain network will tend to stabilize. On the one hand, the supply chain network should improve the awareness of risk prevention, risk response, and risk recovery; shorten the duration of network instability; and maintain the stability of the system and the stability of the nodes themselves, so as to reduce the losses brought by risks to each enterprise in the supply chain and the supply chain as a whole. On the other hand, the elimination of some poorly operated enterprises in the supply chain network is a normal phenomenon, which has a positive effect on the stability and self-adjustment of the whole supply chain network. When maintaining the stability of the supply chain system from the overall perspective, the input and benefit of maintenance cost should be analyzed and judged in conjunction with the actual situation, so as to maximize the overall benefit of the supply chain and avoid meaningless excessive maintenance.

(2) Once a risk occurs, it will spread in a short period of time, causing damage to the supply chain network. Therefore, risk prevention should be given top priority when risk management is carried out by supply chain network node enterprises. Preventing risks, one is to prevent risk generation. Enterprises should analyze and classify the potential risk factors in the supply chain, and they should take targeted measures to prevent risk generation in the process of operation and management according to the characteristics of different types of risks. Second, to prevent themselves from being infected by risks, when contacting other nodes in the supply chain network, enterprises needs previous research and understanding of the node enterprises contacted. Through the results of research and analysis, the potential risk factors in these enterprises should be known, the risk level of the cooperative enterprises should be assessed, the cooperation plan should be reasonably planned, and the contingency plan should be prepared beforehand. Thus, effective response can be made after the unexpected risk event of the cooperative enterprises to avoid the spillover to companies.

(3) When the risk has already started to spread in the supply chain network, each node enterprise in the supply chain network has the obligation and necessity to take measures to control the spread of the risk, regardless of whether it is affected by the risk, in order to restore the stability of the supply chain system as soon as possible. Enterprises that have been infected by the risk should adopt measures to deal with the risk without hesitation or should be eliminated due to their inability to resist the risk. Meanwhile, these enterprises are expected to provide accurate risk-related information to the supply chain network as a whole in a timely manner, so as to provide reference for other nodes to prevent risks and offer help. The nodes in the supply chain network are closely connected in logistics, information flow, and capital flow. Each node in the supply chain network should not fight alone but make common cause while controlling the spread of supply chain risks. Companies that have not yet been infected by the risk should improve their own ability to resist the risk while assisting cooperating infected companies to cope with the risk. Minimizing the time required to control supply chain risks is essential to reduce the damage of supply chain risks to the entire supply chain network.

(4) Establishing immunity to supply chain network risks is the key means to maintain good operation of supply chain node enterprises, restore the stable state of supply chain, remove the subsequent impact of supply chain risks, and prevent risks from arising again. It should be noted that immunization against supply chain risks is not established once and for all, and there is a possibility that immunization may disappear as the internal and external environment of the supply chain network change. The node companies should regularly evaluate the supply chain risk prevention situation to avoid any oversight in the immunization process so as not to leave any future problems. 
In this paper, the mechanism of supply chain risk transmission is studied from a macro perspective. Suggestions for dealing with supply chain risks in the three stages of risk prevention, risk control, and risk immunity are provided in response to the research results. Modeling simulation is used in the research to simulate the impact of risk on supply chain network node companies. The characteristics of the supply chain network risk propagation model are analyzed based on the simulation results. Future research should combine the actual supply chain network; select appropriate parameters; explore the characteristics of risk propagation in the actual supply chain network; and propose targeted supply chain risk prevention methods, control measures, and immunization strategies.

\section{Data Availability}

Some or all data, models, or code that support the findings of this study are available from the corresponding author upon reasonable request.

\section{Conflicts of Interest}

The authors declare that they have no conflicts of interest.

\section{Acknowledgments}

This research was supported by the fund project of the National Natural Science Foundation of China, "Research on Collaborative Control of Urban Logistics Based on Network Theory" (61772062), and the BUCEA Post Graduate Innovation Project (PG2021065).

\section{References}

[1] A. Sharma, A. Adhikary, and S. B. Borah, "Covid-19's impact on supply chain decisions: strategic insights from NASDAQ 100 firms using Twitter data," Journal of Business Research, vol. 117, pp. 443-449, 2020.

[2] B. Lv, J. Han, and X. Fan, "Research on the new mechanism of supply chain risk transmission based on SIR - SCR model under the background of the new technology revolution," Science and Technology Management Research, vol. 40, no. 5, pp. 199-206, 2020, in Chinese.

[3] U. Jüttner, H. Peck, and M. Christopher, "Supply chain risk management: outlining an agenda for future research," International Journal of Logistics: Research and Applications, vol. 6, no. 4, pp. 197-210, 2003.

[4] E. Hassini, S. K. Cheng, and B. H. Kam, "A conceptual framework for analysing risk in supply networks," Journal of Enterprise Information Management, vol. 21, no. 4, pp. 345360, 2008.

[5] G. Cheng and Q. Liu, "Study of supply chain risk transmission model," Journal of Wuhan University of Technology, no. 2, pp. 36-41, 2009, in Chinese.

[6] G. Li, "Research on supply chain risk conduction mechanism," China Business and Market, no. 1, pp. 41-44, 2011, in Chinese.

[7] Z. Li, "Research on supply chain risk identification based on risk conduction mode," China Journal of Commerce, no. 20, pp. 57-59, 2010, in Chinese.
[8] C. Z. Huang and L. Wang, "Research on demand transmission process risk management of supply chain based on fractal theory," in Proceedings of the International Conference on Information Systems for Crisis Response and Management (ISCRAM), pp. 182-186, IEEE, Harbin, China, 2011 November.

[9] G. Cheng and Q. Liu, "To study on changing for transmitting path of the supply chain risk," Value Engineering, no. 4, pp. 1-3, 2009, in Chinese.

[10] C. Shi, J. Xiao, and H. Xue, "Research on the probability of supply chain risk transmission based on bayesian network," Journal of Computational and Theoretical Nanoscience, vol. 13, no. 7, pp. 4158-4162, 2016.

[11] Z. Jiantong, C. Xiaodong, and F. Chencheng, "Transmission of a supplier's disruption risk along the supply chain: a further investigation of the Chinese automotive industry," Production Planning \& Control, vol. 2, pp. 1-17, 2018.

[12] H. H. Chen and A. M. Lin, "Complex network characteristics and invulnerability simulating analysis of supply chain," Journal of Networks, vol. 7, no. 3, 2012.

[13] H. Wang, T. Gu, M. Jin, R. Zhao, and G. Wang, "The complexity measurement and evolution analysis of supply chain network under disruption risks," Chaos, Solitons \& Fractals, vol. 116, pp. 72-78, 2018.

[14] J. Jiang, C. Liu, H. Huang, and H. Zhang, "Risk communication and simulation of supply chain," in Proceedings of the 2017 First International Conference on Electronics Instrumentation \& Information Systems (EIIS), pp. 1-5, IEEE, Harbin, China, 2017 June.

[15] K. Yang and Z. Zhang, "Simulation of SIS-RP model in supply chain network risk propagation," Journal of Beijing Jiaotong University, vol. 37, no. 3, pp. 122-126, 2013, in Chinese.

[16] Q. Jing, "Research on risk conduction of grain supply chain based on sir model," Master's Thesis, Wuhan Polytechnic University, Wuhan, China, 2016, in Chinese.

[17] L. Tang, "Research on logistics risk propagation mechanism of fresh e-commerce supply chain based on sirs model," Master's Thesis, Chongqing University, Chongqing, China, 2019, in Chinese.

[18] J. Wang, H. Zhou, and X. Jin, "Risk transmission in complex supply chain network with multi-drivers," Chaos, Solitons \& Fractals, vol. 143, Article ID 110259, 2021.

[19] K. Yang, "Research on supply chain network risk management based on complex network theory," Doctoral Dissertation, Beijing Jiaotong University, Beijing, China, 2014, in Chinese.

[20] C. X. Liu, T. Shu, S. Y. Wang, S. Chen, and K. K. Lai, "Supply chain disruption risk conduction route based on the small world network," Systems Engineering-Theory \& Practice, vol. 35, no. 3, pp. 608-615, 2015, in Chinese. 